\title{
Network high-performance grinding devices for ferromagnetic materials
}

\author{
Yuriy Vernigorov ${ }^{1 *}$, Valeriy Lebedev ${ }^{1}$, Natalya Frolova ${ }^{1}$, and Kirill Leletko ${ }^{1}$ \\ ${ }^{1}$ Don State Technical University, Gagarin sq., 1, Rostov-on-Don, 344000, Russia
}

\begin{abstract}
The design features of the grinding devices that implement the impact destruction of the ferromagnetic materials particles in a magneto vibrating layer formed in a non-uniform magnetic field are considered. It is shown that when a magnetizable powder is affected by an alternating magnetic field with certain parameters, a magneto vibrating layer is formed, under the conditions of which, a random perturbing factor occurs. It is caused by the dipole particles - clusters interactions and provides highefficient finish powder grinding. Methods for producing metal powders, which are distinguished according to the operating principle and to the requirements for the technological properties of the powders obtained, are analyzed. For coarse grinding, jaw, roller and cone crushers and mullers are used; at this, particles of 1-10 $\mathrm{mm}$ in size, which are the source material for fine grinding, are obtained. The finish grinding of the material obtained is carried out on the ball rotating, vibrating or planar centrifugal, vortex and hammer mills. The main drawback of these techniques of metal powder grinding is sticking of grinding body residue on the powder particles, which reduces the quality and operational properties of the powder. A relation to calculate the dependence of the fineness number of ferromagnetic materials on the induction gradient of an external variable magnetic field is proposed. The design features of an electromagnetic mill based on a screw drum that, due to the spatial orientation of its walls, ensures an effective movement of powder flows inside it, such as mixing, rotation, oncoming movement, translational motion and simultaneous advancement through the drum are presented. The concept and technological options of grinding powders in an electromagnetic mobile hammer mill are revealed, which enables to obtain a powder of a given particle size distribution with high uniformity. It is established that mills in which a magneto vibrating layer is implemented are more effective than mechanical ones: grinding of ferromagnetic powders in a magneto vibrating layer increases drastically the performance of the grinding process. Changing the parameters of the electromagnetic field, you can set an average particle size and the degree of homogeneity of the powder.
\end{abstract}

\section{Introduction}

The specific properties of disperse materials allow for the use of powder products in virtually every area of engineering: nuclear energy, space technology, automobile

\footnotetext{
*Corresponding author: jvernigorov@dstu.edu.ru
} 
production, etc. Many technologies for producing powders of ferromagnetic materials contain operations of separation, homogenization, fractionation, high-temperature annealing, surface alloying, etc. The intensification of operations is possible, for ex-ample, through creating a vibrating layer from a dispersed medium. However, none of the conventional methods for producing a vibrating layer can provide spatial stability of a polydisperse medium with high porosity of particles with a size of $1-500$ microns. In the disperse heterogeneous systems of ferromagnetic particles in variable and rotating electromagnetic fields, a magneto vibrating layer (MVL) is formed. The most characteristic spatially stable state of particles and aggregates in the MVL are magneto fluidization (magnetic boiling) and a volume-textured system of vibrating aggregates.

\section{Efficiency of grinding particles in a magneto vibrating layer}

Methods for producing metal powders can be divided into two main types: physicalmechanical and chemical-metallurgical ones. Milling devices differ in the operating principle and the requirements for the technological properties of the powders obtained [1]. For coarse grinding, jaw, roller and cone crushers and mullers are used; at that, particles of $1-10 \mathrm{~mm}$ in size, which are the source material for fine grinding, are obtained. The finish grinding of the material obtained is carried out on the ball rotating, vibrating or planar centrifugal, vortex and hammer mills. Attrition devices are among the most energyintensive milling units and, in addition to grinding, are used to obtain powders through mechanical alloying. In attritors, the required dispersion of powders is obtained several times faster than in drum mills. In planetary centrifugal mills, the material is milled by grinding bodies disposed in the form of a segment and moving together with the drum. The main drawback of the specified techniques of milling metal powders is sticking of grinding body residue on the powder particles, which reduces the quality and operational properties of the powder.

Another class of mills that do not contain grinding bodies, in which grinding material does not adhere to powder particles, are jet, jet-vortex and ultrasonic devices. In jet mills, the grinding of the material is ensured by the energy transferred to its particles through the jets of compressed gas or superheated steam. The mill feed in the chamber is in the fluidized layer. In vortex mills, grinding is carried out by propellers or hammers that are disposed in the working chamber and are counterrotating at high (about 3,000 rpm) speeds. Under ultrasonic grinding, high-frequency waves affect the suspension of powder in a liquid. Propagating elastic vibrations create gage pressure zones and fluid discontinuities cavitation bubbles. When riveting, these bubbles form shock waves sufficient to overcome the strength of solid materials.

When a ferromagnetic powder is affected by a variable magnetic field with certain parameters, a MVL is formed [3, 10]. The motion of particles in the state of a developed MVL is complicatedly dependent on the properties of the ferromagnetic material, the field topology, and is determined by the combined action of a large number of heterogeneous physical factors, which hampers the selection of field parameters that ensure the establishment of optimal application modes. In the magneto vibrating layer there is a random perturbing factor caused by the dipole interactions of particles and clusters. An abrupt change in the initial conditions determined by collisions with other particles and with the walls of the chamber is also possible.

Ferromagnetic materials with high interparticle interaction forces require the use of purpose-built procedures under milling. Many ferromagnetic materials are pyrophoric, which also requires additional protective measures under grinding. Techniques of powder 
fine grinding based on the applied shock on the mill feed are widespread in powder metallurgy.

Consider a polydisperse medium of a ferromagnetic material. Suppose that particles of a medium are spheres with frozen-in magnetic moments placed in an external variable magnetic field whose topology is such that its energy is transferred to particles of a disperse medium mainly to the translational degrees of freedom [3].

With regard to the assumptions made, equalizing the energy transferred to the particles from the magnetic field and the destruction energy of a spherical particle according to the hypothesis of V. L. Kirpichev [4], we determine the fineness number - field induction gradient dependence:

$$
Z_{u}=\sqrt[3]{\frac{1}{1-A\left(\frac{\partial B_{y}}{\partial y}\right)^{2}}}
$$

where

$$
A=\frac{E J^{2}}{\rho \omega^{2} \sigma^{2}}
$$

is a constant dependent on the mechanical and magnetic properties of the material and the frequency of the variable field; $\sigma_{\text {is the tensile strength }}$ upon destruction of the material; $\mathrm{d} \kappa$ is the final particle diameter; $Z_{\mathcal{u}}=\frac{d_{H}}{d_{\kappa}}$ is the material fineness number; $\mathrm{E}$ is the Young's modulus of the material; $\rho$ is the density of the material; $\partial B_{v} / \partial y$ is the field induction gradient; $\omega$ is the frequency of the variable magnetic field; $\mathrm{J}$ is the magnetization of the material.

When deriving (1), it is assumed that the dimensions of the working volume of the milling device are such that the process of particle interaction with the walls occurs each half of the period, i.e. $T / 2=\pi / \omega$.

The relation (1) allows us to calculate the dependence of the fineness number of ferromagnetic materials on the induction gradient of an external variable magnetic field.

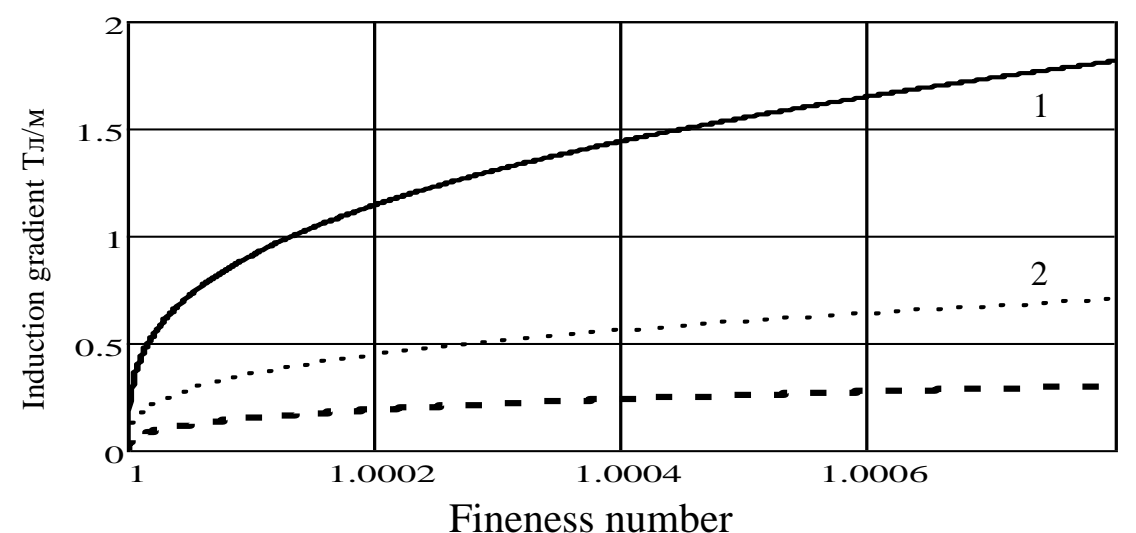

Fig. 1. Gradient of magnetic field induction providing a given fineness number for various materials: $1-\mathrm{SmCo}_{5} ; 2-\mathrm{NdFeB} ; 3-\mathrm{Ba}_{6} \mathrm{Fe}_{12} \mathrm{O}_{19}$ 


\section{$3 \quad$ Helical electromagnetic mill}

Fig. 2 shows an impact helical mill, which principle is based on the use of a magneto vibrating layer.

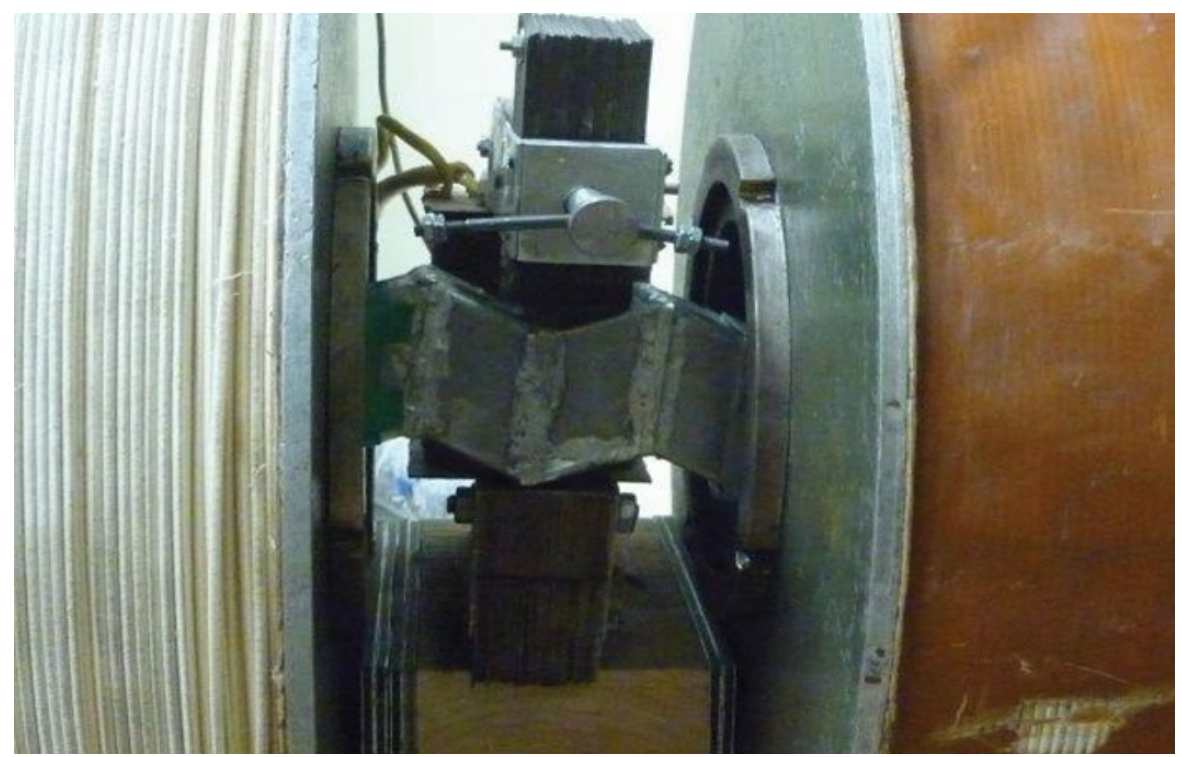

Fig. 2. View of interpole space of impact mill

Using a screw drum (Fig. 3), due to the spatial orientation of its walls, it is possible to create a movement of powder flows inside it, so that mixing, rotation, oncoming movement, translational motion and simultaneous advancement through the drum will occur $[6,8]$. The particle size distributions of SmCo5 powder are shown in Fig. 4.
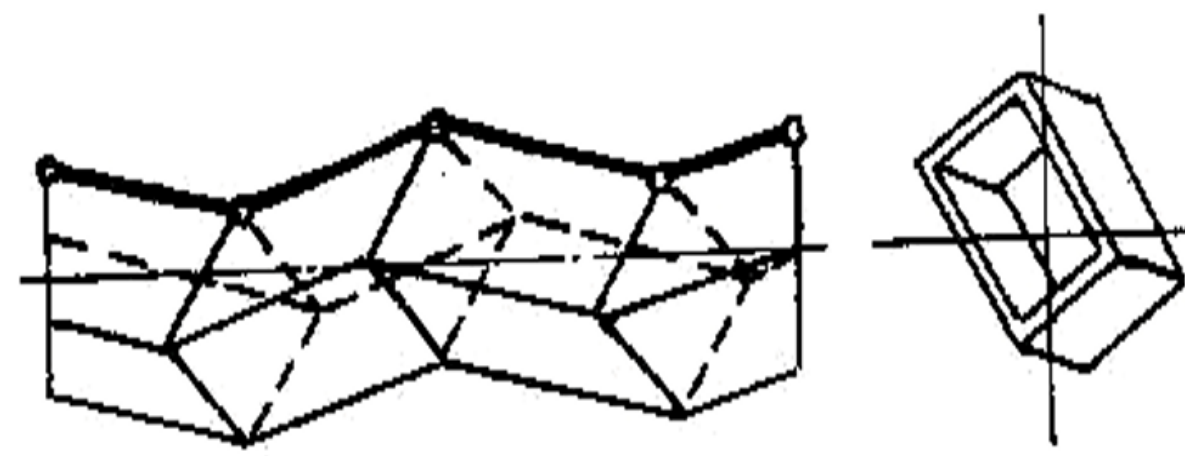

Fig. 2. Screw drum model 

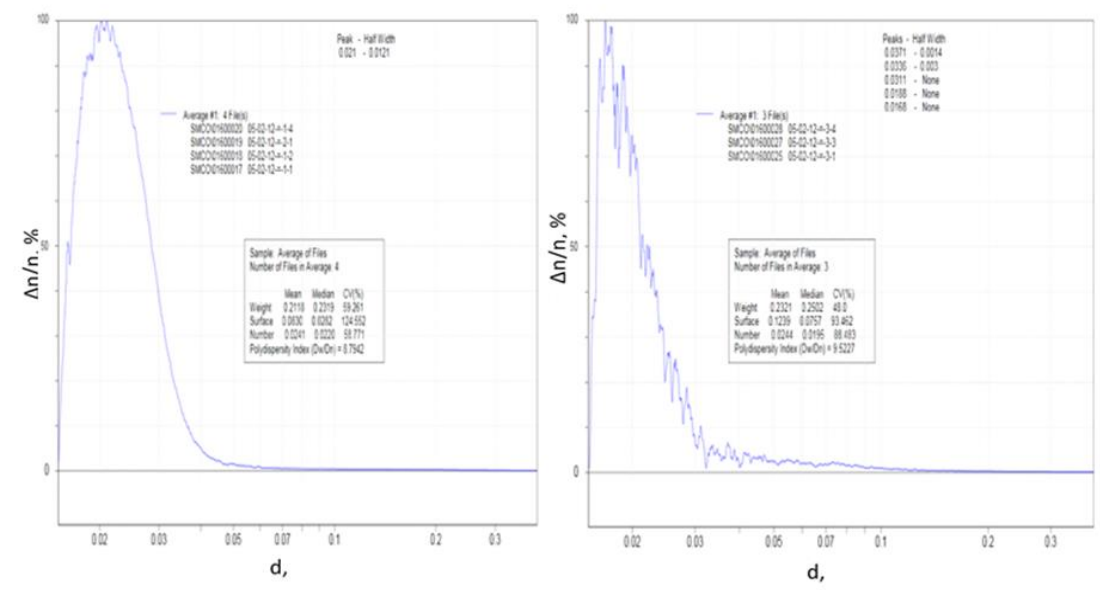

Fig. 4. SmCo5 particle size distribution under grinding in helical electromagnetic mill during milling time: a - 40 minutes, $\mathrm{b}$ - 60 minutes

\section{Electromagnetic hammer mill}

A particular place in a number of milling devices providing dry grinding is occupied by a mill, in which a disperse medium of ferromagnetic material forms a magneto vibrating layer. Grinding of the ferromagnetic material is performed by rotating hammers. The device enables to obtain a powder of a given particle size distribution with high uniformity [7].

The mill (Fig. 5) contains a cylindrical working chamber through which the shaft driven by an electric motor with a frequency of 15,000 rpm passes axially. The lower end of the shaft is equipped with hammers arranged in two rows, which are oriented perpendicular to each other. The working chamber housing is provided with a hole for supplying inert gas.

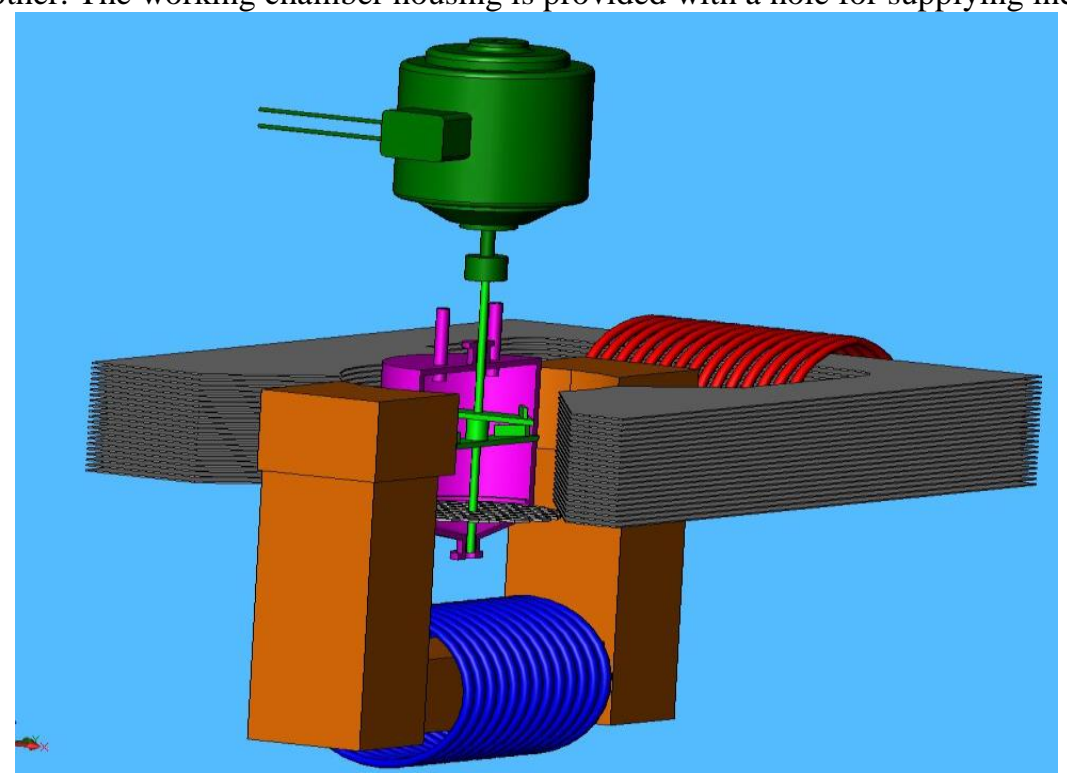

Fig. 3. Hammer mill model 
To create a constant magnetic field, an electromagnet containing two polar tips with a diameter of $25 \mathrm{~mm}$ with an adjustable interpole gap, a magnetic circuit and two coils with 3,000 turns of the PBO brand each, with $2 \mathrm{~mm}$ wire diameter was used. A working chamber made of optical glass with a diameter of $60 \mathrm{~mm}$ and a height of $60 \mathrm{~mm}$ was placed in the generic interpole space of electromagnets.

To create a variable inhomogeneous magnetic field, a cut-off permeameter was used, consisting of two coils containing 500 turns of PBO wire each and an imbricated core made of electrical tape. The induction gradient of a variable magnetic field was determined using the e.m.f. measured by an inductive sensor.

The inductive sensor is made in the form of a cylindrical coil having 200 turns of PEL-2 brand wire wound around a plastic frame $4 \mathrm{~mm}$ high, with an internal diameter of $2 \mathrm{~mm}$ and an external diameter of $8 \mathrm{~mm}$. The signal induced in the sensor was measured with a B3-57 voltmeter.

The signal induced in the sensor was measured with the B3-57 voltmeter. The values of the e.m.f. induced in the inductive sensor were measured on the axis of the interpole space at a distance of $10 \mathrm{~mm}$ from one pole, at a distance of $20 \mathrm{~mm}$, at a distance of $30 \mathrm{~mm}$ and at the midpoint of the interpole space. A general view of the pilot plant is shown in Fig. 6.

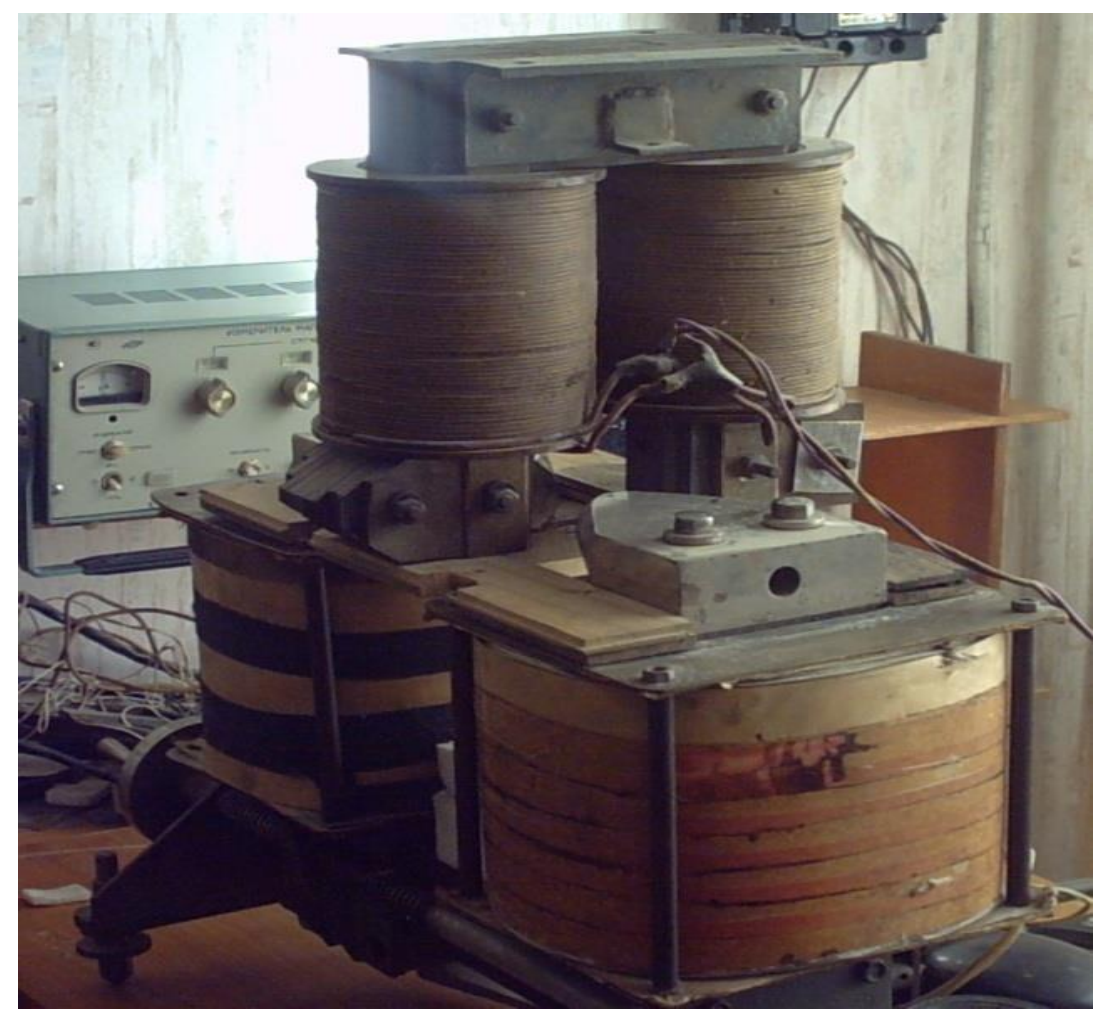

Fig. 4. View of laboratory hammer mill

The calibration curves of $\partial B_{v} / \partial y=f(I)$ AC electromagnet at the distance between the electrodes of $5 \mathrm{~cm}$ are shown in Fig.7. 


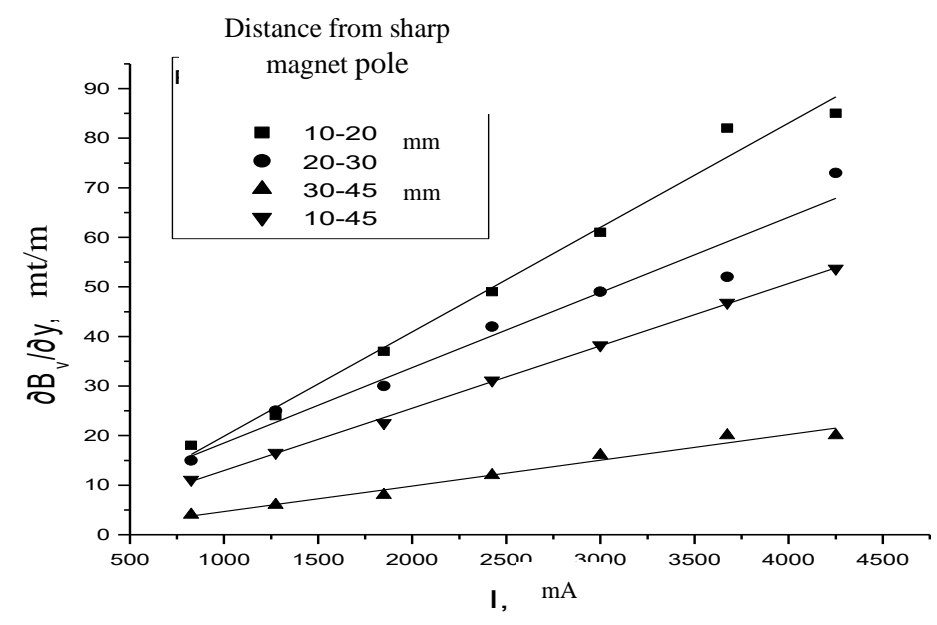

Fig. 5. Dependence of induction gradient variable magnetic field in inter-pole space of electromagnet on the current

The dependences of the average particle size, median and dispersion of the $\mathrm{Nd}-\mathrm{Fe}-\mathrm{B}$ alloy powder on the milling time are shown in Fig. 8.

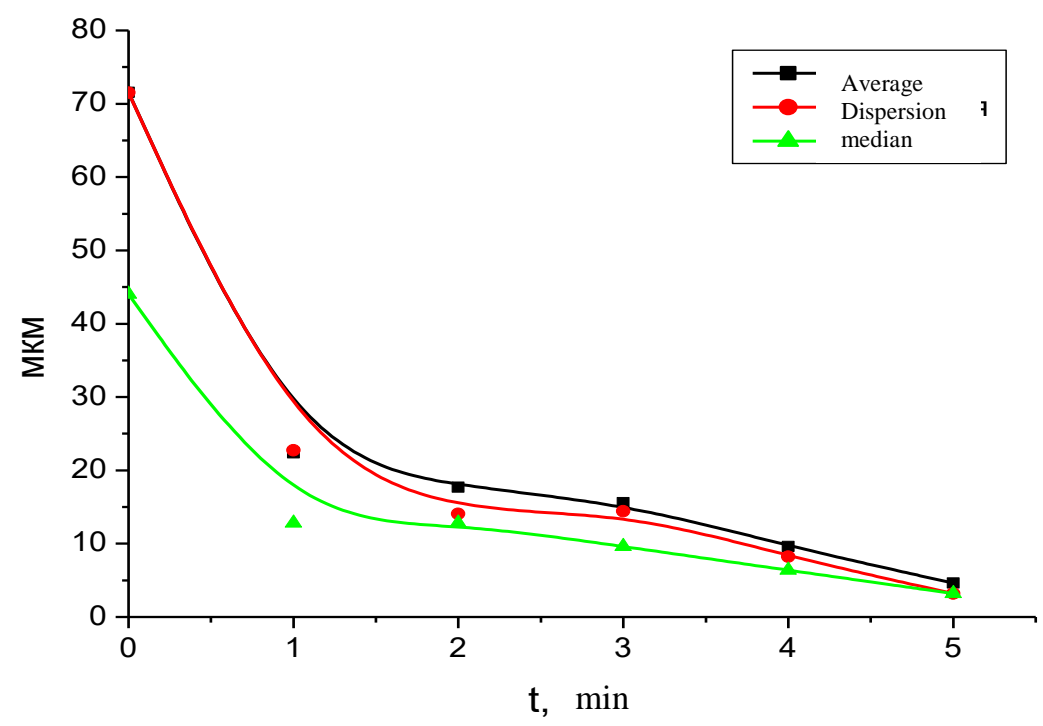

Fig. 6. Dependences of average particle size, median, and powder dispersion based on Nd-Fe-B alloy on milling time in variable magnetic field with induction gradient of $126.6 \mathrm{mT} / \mathrm{m}$

The probability curves of the particle size distribution of the Nd-Fe-B alloy powder by different grinding time periods in a hammer mill in a magneto vibrating layer formed in a magnetic field with the induction gradient of $126.6 \mathrm{mT} / \mathrm{m}$ are shown in Fig. 9. 


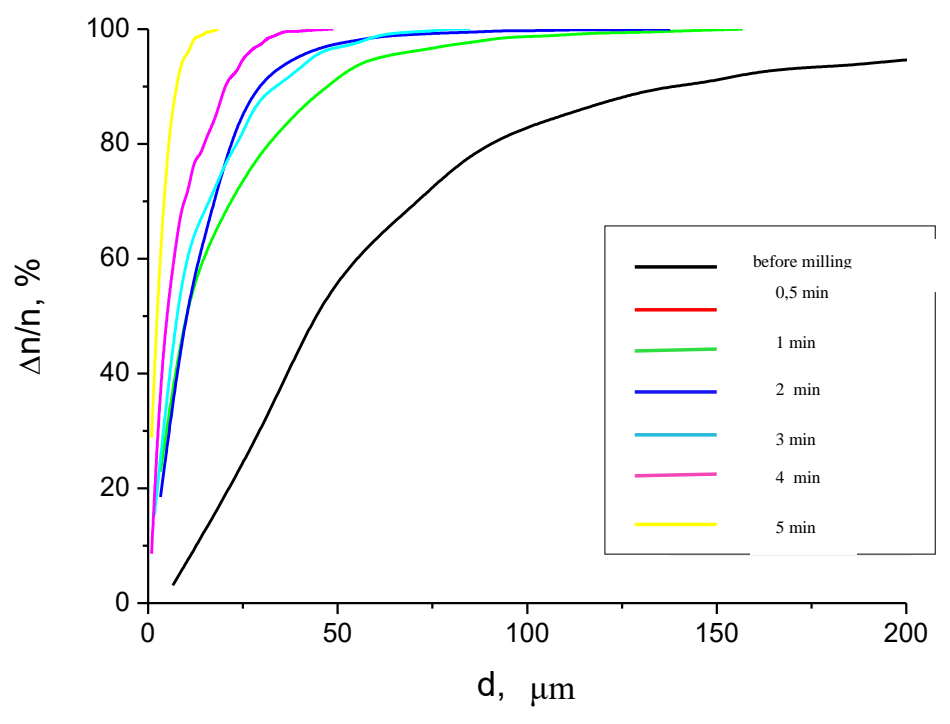

Fig. 7. Cumulative probability curves before milling and under milling in variable magnetic field with induction gradient of $126.6 \mathrm{mT} / \mathrm{m}$

\section{Conclusion}

Mills, in which operation a magneto vibrating layer is implemented, are more effec-tive than mechanical ones - grinding of ferromagnetic powders in a magneto vibrat-ing layer increases drastically the processing rate. Changing the parameters of the electromagnetic field, you can set an average particle size and the degree of homoge-neity of the powder.

The destruction of particles in the proposed designs of the mills occurs both due to collisions with hammers and walls of the mill, and due to interparticle encounters. Autogenous milling of the powder is associated not with the destruction of aggregates, but with the crushing of particles.

The advantages of the designs under consideration are as follows: milling in a protective environment; control of the powder particle size distribution using an electromagnetic field. The disadvantages include grinding of ferromagnetic materials only; the presence of mechanically rotating structural elements in the mill, which increase energy costs and wear of mechanisms.

\section{References}

1. G.A. Libenson, V.Yu. Lopatin, G.V. Komarnitsky, MISIS, 368 p. (2001).

2. A.A. Garabazhiu, E.I. Levdansky, A.E. Levdansky, Proceedings of the National Academy of Sciences of Belarus. Physics and Mathematics Series, 2, 125-131 (2000).

3. Yu.M. Vernigorov, I.N. Egorov, S.I. Egorova, Journal of Magnetism and Magnetic Materials, 290-291, 1177-1180 (2005).

4. V.L. Kirpichev, $5^{\text {th }}$ edition: Moscow, Leningrad “Gostekhizdat", 360 p. (1951).

5. Yu.M. Vernigorov, N.N. Frolova, Vestnik of DSTU. 11, 7 (58), 1127-1131 (2011). 
6. Yu.M. Vernigorov, et al., Proc. Int. Sci.-Pract. Conf., Technical Sciences, 5, 52-56.

7. V.A. Lebedev, Yu.M. Vernigorov, A.A. Kochubey, Progressive Technologies and Systems of Mechanical Engineering. Donetsk National Technical University. 1(52), 84-91 (2016).

8. V.A. Lebedev, G.V. Serga, M.M. Chaava, G.V. Demin, Science Intensive Technologies in Mechanical Engineering. 10 (76), 24-27 (2017).

9. V.A. Lebedev, A.A. Kochubey, A.V. Kiricheck, IOP conference series: Materials Science and Engineering. 10. Ser. "International Conference on Mechanical Engineering, Automation and Control Systems 2016", (2017).

10. S.I. Egorova, Rostov-on-Don, 162 p. (2009). 